Review began 11/07/2021 Review ended 11/15/2021 Published 11/17/2021

(c) Copyright 2021

Ali et al. This is an open access article distributed under the terms of the Creative Commons Attribution License CC-BY 4.0., which permits unrestricted use, distribution, and reproduction in any medium, provided the original author and source are credited.

\section{Systemic Lupus Erythematosus With Hemophagocytic Lymphohistiocytosis: Is COVID- 19 the Inciting Factor?}

\author{
Ruhma Ali ${ }^{1}$, Rime Mehannek ${ }^{1}$, Aditya Patel ${ }^{1}$, Amy Paige ${ }^{1}$, Sahithi Reddy ${ }^{2}$, Michael Guma ${ }^{3}$, Gunwant \\ Guron $^{2}$ \\ 1. Internal Medicine, Saint Michael's Medical Center, Newark, USA 2. Hematology and Oncology, Saint Michael's \\ Medical Center, Newark, USA 3. Rheumatology, Saint Michael's Medical Center, Newark, USA
}

Corresponding author: Ruhma Ali, raoruhma@gmail.com

Categories: Infectious Disease, Rheumatology, Hematology

Keywords: autoimmune disease, cytokine storm, covid-19, hemophagocytic lymphohistiocytosis, systemic lupus erythematosus

\title{
Introduction
}

Hemophagocytic lymphohistiocytosis (HLH) is an underrecognized, life-threatening condition that requires prompt treatment and diagnosis. HLH is categorized into two separate forms. The primary or familial form is autosomal recessive [1], whereas the acquired or secondary form can be due to infectious causes, malignancies, or rheumatological disorders. HLH is characterized by systemic hyperinflammation due to the uncontrolled proliferation of lymphocytes, macrophages, and CD8 T-cells, leading to massive cytokine release. This sequence of events leads to tissue damage and progressive multiorgan failure [2]. The hallmark features of HLH include persistent fevers, pancytopenia, hepatosplenomegaly, hyperferritinemia, and hypertriglyceridemia. HLH in the background of systemic lupus erythematosus (SLE) is uncommon and requires clinical acuity and awareness to avoid complications from the sequelae of the disease. Viral infections are known to trigger secondary HLH. The clinical and laboratory features of secondary HLH and coronavirus disease 2019 (COVID-19) are synonymous, with COVID-19 likely serving as a nidus for unmasking autoimmune diseases.

\section{Case Presentation}

A 25-year-old female with a medical history of asthma and depression presented to the Emergency Room with chief complaints of generalized weakness, fatigue, nausea, vomiting, and bloody diarrhea that progressively worsened in the past two months. She had presented to the Emergency Department (ED) two months back for a cough and shortness of breath. At that time, she denied any headache, neck stiffness or pain, sore throat, or chest pain. Her physical examination was normal. Reverse transcription-polymerase chain reaction for severe acute respiratory distress syndrome coronavirus 2 (SARS-CoV-2) was positive. Ddimer was also markedly elevated. Chest X-ray showed no cardiopulmonary abnormality. She did not require hospitalization and was told to home quarantine for two weeks. She did not receive remdesivir,

dexamethasone, or anticoagulation for COVID-19. However, she received oseltamivir for five days as she tested positive for the influenza B virus. At the time of the current admission, she complained of a rash on her face and scalp as well as swelling of her tongue and lips. The patient stated that she has been restricted to the bed due to severe muscle weakness and had become irritable and anxious. Her family members noticed a change in her affect and concentration. She acknowledged taking pain medications without any significant relief and denied smoking or alcohol use. A review of the others systems was unremarkable. On admission, she was afebrile, with a blood pressure of $130 / 80 \mathrm{mmHg}$, respiratory rate of 28 breaths/minute, heart rate of 101 beats/minute, oxygen saturation of $95 \%$ on room air, and body mass index of $28.52 \mathrm{~kg} / \mathrm{m}^{2}$. On physical examination, the patient appeared weak and tired. She had pale conjunctiva with jaundice. Tenderness was noted on palpation of the sternum and left chest. Abdominal examination was significant for mild epigastric tenderness. Additionally, right inguinal lymphadenopathy was noted. Muscle strength 


\section{Cureus}

was 3/5 in bilateral lower extremities and 4/5 in bilateral upper extremities. Cranial nerves were grossly intact; however, knee reflexes were not appreciated. A few dry, brownish lesions of 3-5 mm diameter were scattered around the nose. Ulcers were noted on the hard palate, measuring approximately $2 \mathrm{~cm}$ in diameter, and on the scalp. Perioral ulceration was also seen.

Initial laboratory investigations revealed elevated triglycerides, lactate dehydrogenase (LDH), fibrinogen, ferritin, and D-dimer with normal C-reactive protein (CRP). Detailed laboratory parameters comparing laboratory findings from the previous ED visit and over the course of hospital stay are listed in Table 1 . She had an acute kidney injury with increased creatinine and rhabdomyolysis with total creatine kinase of 3,223 U/L (reference range: 38-176 U/L). Her chest X-ray showed a possible consolidation with pleural effusion. An echocardiogram showed large pericardial effusion. Diagnostic pericardiocentesis was done which was consistent with transudative pericardial fluid from ongoing underlying inflammatory conditions.

\begin{tabular}{|c|c|c|c|c|c|c|}
\hline $\begin{array}{l}\text { Laboratory } \\
\text { parameters }\end{array}$ & $\begin{array}{l}\text { Previous ED visit (2 } \\
\text { months prior) }\end{array}$ & $\begin{array}{l}\text { Admission } \\
\text { day } 1\end{array}$ & $\begin{array}{l}\text { In-hospital } \\
\text { day } 7\end{array}$ & $\begin{array}{l}\text { Extubation } \\
\text { (day 53) }\end{array}$ & $\begin{array}{l}\text { 1-week post-extubation } \\
\text { (day } 60 \text { ) }\end{array}$ & $\begin{array}{l}\text { Reference } \\
\text { range }\end{array}$ \\
\hline Hemoglobin (g/dL) & 10.4 & 10.2 & 8.1 & 8.8 & 8.2 & $12-15.5$ \\
\hline $\begin{array}{l}\text { Neutrophils } \\
\left(\times 10^{3} / \mu \mathrm{L}\right)\end{array}$ & 1.9 & 1.3 & 11.1 & 4.6 & 3.9 & $1.7-7$ \\
\hline $\begin{array}{l}\text { Lymphocytes } \\
\left(\times 10^{3} / \mu \mathrm{L}\right)\end{array}$ & 0.8 & 2.7 & 0.4 & 1.3 & 1.4 & $0.9-2.9$ \\
\hline $\begin{array}{l}\text { Platelet count } \\
\left(\times 10^{3} / \mu \mathrm{L}\right)\end{array}$ & 109 & 35 & 60 & 92 & 126 & $150-450$ \\
\hline Creatinine (mg/dL) & 0.7 & 2.4 & 5 & $<0.2$ & 0.2 & $0.6-1.2$ \\
\hline CRP (mg/dL) & 0.6 & $<0.3$ & & 0.9 & & $0.0-0.8$ \\
\hline Ferritin (ng/mL) & 143.6 & 17,500 & 960.2 & & & $11-307$ \\
\hline Bilirubin (mg/dL) & 0.3 & 5.7 & 3.9 & 0.5 & 0.3 & $0.2-1.2$ \\
\hline $\mathrm{LDH}(\mathrm{U} / \mathrm{L})$ & 307 & 2252 & 1145 & 405 & 333 & $122-222$ \\
\hline Fibrinogen (mg/dL) & & 82 & 259 & 169 & 186 & 200-393 \\
\hline $\begin{array}{l}\text { Triglyceride } \\
(\mathrm{mg} / \mathrm{dL})\end{array}$ & 142 & 405 & 431 & & & $0-150$ \\
\hline D-dimer (ng/mL) & 1891 & 7086 & 7280 & & & $0-500$ \\
\hline AST (U/L) & 35 & 3831 & 456 & 23 & 14 & 10-36 \\
\hline ALT (U/L) & 18 & 535 & 52 & 35 & 24 & $6-29$ \\
\hline
\end{tabular}

\section{TABLE 1: Laboratory parameters.}

ED: Emergency Department; CRP: C-reactive protein; LDH: lactate dehydrogenase; AST: aspartate transaminase; ALT: alanine transaminase

The patient was admitted to the Intensive Care Unit for close monitoring and further investigation. She had two episodes of seizure with altered mental status and was intubated for airway protection. The initial bicytopenia evolved to pancytopenia, with a white blood cell count of $3.9 \times 10^{3} / \mu \mathrm{L}$, hemoglobin $6.8 \mathrm{~g} / \mathrm{dL}$, and platelets $35 \times 10^{3} / \mu \mathrm{L}$. Her renal function continued to decline with a peak creatinine of $5.5 \mathrm{mg} / \mathrm{dL}$, and she was started on hemodialysis. Her initial complement level (C3 and C4) counts were low, and she had multiple positive antibodies, including anti-double-stranded DNA, anti-Smith, anti-ribonucleoprotein, antiRo, and anti-La. The rheumatologic workup is shown in Tables 2, 3. A diagnosis of SLE was supported by the skin and hard palate ulcerations, pleuropericardial disease, and immunological workup. A presumptive diagnosis of HLH was supported by hyperferritinemia, hypertriglyceridemia, pancytopenia, lymphadenopathy, and severe hepatic inflammation. The initial HScore for reactive hemophagocytic syndrome showed a $70-80 \%$ probability of HLH with a total of 187 points. 


\section{Cureus}

\begin{tabular}{|c|c|c|c|c|}
\hline Antibodies & Day 4 & Day 33 & Day 72 & Reference range \\
\hline SSA & $>8.0$ & $>8.0$ & - & $0.0-0.9$ \\
\hline SSB & 2.8 & 0.6 & - & $0.0-0.9$ \\
\hline Rho factor (IU/mL) & $<0.10$ & - & - & $0-15$ \\
\hline Anti-Jo & $<0.2$ & $<0.2$ & - & $0.0-0.9$ \\
\hline Anti-DNA (IU/mL) & 251 & 235 & 10 & $0-9$ \\
\hline Anti-Smith & $>8.0$ & $>8.0$ & - & $0.0-0.9$ \\
\hline B2 microglobulin (mg/L) & 17.2 & - & - & $0.6-2.4$ \\
\hline
\end{tabular}

TABLE 2: Rheumatological parameters in the hospital.

SSA: anti-Ro; SSB: anti-La

\begin{tabular}{|c|c|c|c|c|c|c|c|}
\hline Complement & Day 1 & Day 3 & Day 24 & Day 32 & Day 67 & Day 70 & Reference range \\
\hline C3 (mg/dL) & $<16$ & 34 & 76 & 56 & 96 & 75 & $75-175$ \\
\hline C4 (mg/dL) & - & $<6$ & 9 & 7 & 17 & 18 & $14-40$ \\
\hline
\end{tabular}

TABLE 3: Complement parameters in the hospital.

Rheumatology, Hematology/Oncology, Gastroenterology, Neurology, Cardiology, and Nephrology departments were consulted. She was started on high-dose pulse steroid therapy with immunosuppressive agents, including mycophenolate mofetil $250 \mathrm{mg}$ daily and hydroxychloroquine $400 \mathrm{mg}$ daily. She also received 14 sessions of plasmapheresis and $600 \mathrm{mg}$ of rituximab twice with improvement in her clinical function. She was monitored for signs of infection while on an increased dose of steroids. During the course of her ICU stay, her mental status improved. Her renal function subsequently normalized and dialysis was stopped. The cytopenia resolved and the inflammatory indices including liver function tests and ferritin gradually returned to normal. The patient was weaned off the ventilator and a trach collar was placed. She was transferred to a long-term acute care facility for rehabilitation of muscle strength.

\section{Discussion}

HLH is a rarely diagnosed, life-threatening condition that consists of a constellation of symptoms caused by cytokine storm and excessive immune activation [1]. The incidence of HLH has been reported to be one case per million persons per year [2]. HLH can occur as the familial primary form due to genetic defects leading to a decrease in the cytotoxic activity of natural killer (NK) cells or due to secondary etiologies, including infection, an autoimmune condition, malignancy, medications, and rheumatological conditions [1]. It usually affects children and young adults. HLH is characterized by immune dysregulation causing uncontrolled proliferation and activation of CD8 T-cells, lymphocytes, and macrophages, which causes hypersecretion of inflammatory cytokines [3]. The cardinal features include clinical and laboratory abnormalities, and consist of high-grade fever, hepatosplenomegaly, cytopenia (anemia, thrombocytopenia, and neutropenia), hyperferritinemia, hypertriglyceridemia, hypofibrinogenemia, reduced or absent NK cell activity, rapidly progressing multiorgan failure, and evidence of hemophagocytosis in the bone marrow [4].

HLH secondary to SLE is a rare clinical entity with an estimated prevalence of 0.9-4.6\% [5]. The diagnosis of HLH secondary to SLE is complicated because they both have overlapping symptoms; however, HLH presents with features of hyperferritinemia, hypofibrinogenemia, and hypertriglyceridemia that are not present in SLE. Another aspect to consider is that HLH might lead to a delay in the diagnosis of SLE; hence, prompt immunological testing for SLE is advised in the setting of HLH to prevent diagnostic delay [2]. It has been reported that viruses may be implicated in the etiology of SLE. The phenomenon of molecular mimicry in which immune reactions against viral antigens may turn against the self-antigens leading to auto-immunity might be the cause of this association [6]. Limited data are available on the onset of clinical manifestations of SLE after infection with SARS-CoV-2; however, several studies have linked COVID-19 with other autoimmune diseases, including rheumatoid arthritis (RA) and multiple sclerosis. Joo et al. observed that viral infections including SARS-CoV-2 can increase the incidence of RA [7]. Tang et al. also reported a case of HLH after receiving a COVID-19 vaccine [8]. Autoantibodies, which are the hallmark of most autoimmune 
diseases, may also be detected in COVID-19 [9]. It has been speculated that SARS-CoV-2 can cause crossreactivity with host cells which might trigger an autoimmune response [10]. SARS-CoV-2 increases tumor necrosis factor-alpha, interferon-gamma, interleukin (IL)-2, 6, 7, and 10, which show a form of secondary HLH [6]. Some of the clinical characteristics and laboratory findings observed in COVID-19 are reminiscent of HLH, including fever, lymphocytopenia, elevated LDH, D-dimer, ferritin, and rapidly progressing multiorgan failure. The cytokine profile observed in COVID-19 is also similar to HLH, with elevated levels of IL-2, 6, and 7 [4].

In our case, the patient presented with features of SLE after a few weeks of COVID-19 diagnosis. The patient was diagnosed with SLE based on the symptoms and immunological workup. As per the HLH-2008 diagnostic criteria, the diagnosis of HLH can be made by either fulfilling five out of eight diagnostic criteria or by molecular identification of HLH-associated gene mutation. In our case, the patient was diagnosed with HLH as she fulfilled five diagnostic criteria [11]. Most recently, a well-validated scoring system for the diagnosis of primary HLH was published, the HScore [12]. This scoring system incorporates cytopenia, fever, organomegaly, fibrinogen, ferritin, alanine aminotransferase, and hemophagocytosis, with an HScore of $>250$ consistent with $99 \%$ probability of HLH and $<90$ conferring $<1 \%$ probability of HLH. The typical treatment for primary HLH includes etoposide, dexamethasone, cyclosporine, and the consideration of hematopoietic stem cell transplant [1]. The treatment of secondary HLH relies on the treatment of the primary disease. Literature has shown that rituximab may be an effective treatment option as an adjunct or monotherapy of HLH, especially in the setting of SLE by modulation of various parts of the cytokine cascade; however, further investigation needs to be done on the use of rituximab in HLH [13].

In the case of a diagnosis of HLH in a patient with COVID-19, it is empirical to control the hyperimmune response that leads to multiorgan failure and death. The effectiveness of the therapy is time-dependent to control the hyperactivated immune system. Corticosteroids, preferably dexamethasone, are the initial choice of treatment; however, plasma exchange can also be performed to eliminate cytokines and improve coagulation state [4]. If there is no improvement of symptoms in a patient with COVID-19, early goaldirected therapy might lead to improved outcomes [14]. A stepwise therapeutic approach with corticosteroids and immunosuppressive therapy might be required to reduce the effects of the cytokine storm and prevent the high mortality risk associated with HLH. Management by a multidisciplinary team, including a rheumatologist, intensivist, hematologist, and immunologist, might be needed to provide the patient with a full range of treatment options.

\section{Conclusions}

Although single case reports have limitations, the present case shows a possible association of COVID-19 with the initial manifestation of SLE. Secondary HLH should be suspected in a patient who meets the diagnostic criteria of HLH. Early diagnosis and prompt intervention are required to reduce the mortality rate in patients in HLH. Further case reports are required to support this hypothesis.

\section{Additional Information \\ Disclosures}

Human subjects: Consent was obtained or waived by all participants in this study. Conflicts of interest: In compliance with the ICMJE uniform disclosure form, all authors declare the following: Payment/services info: All authors have declared that no financial support was received from any organization for the submitted work. Financial relationships: All authors have declared that they have no financial relationships at present or within the previous three years with any organizations that might have an interest in the submitted work. Other relationships: All authors have declared that there are no other relationships or activities that could appear to have influenced the submitted work.

\section{References}

1. Thornton CS, Minoo P, Schneider M, Fifi-Mah A: Severe skin disease in lupus associated with hemophagocytic lymphohistiocytosis: case reports and review of the literature. BMC Rheumatol. 2019, 3:7. 10.1186/s41927-019-0055-x

2. Rahal AK, Fernandez J, Dakhil C: Undiagnosed systemic lupus erythematosus presenting as hemophagocytic lymphohistiocytosis. Case Rep Rheumatol. 2015, 2015:748713. 10.1155/2015/748713

3. Henter JI, Elinder G, Söder O, Ost A: Incidence in Sweden and clinical features of familial hemophagocytic lymphohistiocytosis. Acta Paediatr Scand. 1991, 80:428-35. 10.1111/j.1651-2227.1991.tb11878.x

4. Opoka-Winiarska V, Grywalska E, Roliński J: Could hemophagocytic lymphohistiocytosis be the core issue of severe COVID-19 cases?. BMC Med. 2020, 18:214. 10.1186/s12916-020-01682-y

5. Gupta D, Mohanty S, Thakral D, Bagga A, Wig N, Mitra DK: Unusual association of hemophagocytic lymphohistiocytosis in systemic lupus erythematosus: cases reported at tertiary care center. Am J Case Rep. 2016, 17:739-44. 10.12659/ajcr.899433

6. Zamani B, Moeini Taba SM, Shayestehpour M: Systemic lupus erythematosus manifestation following COVID-19: a case report. J Med Case Rep. 2021, 15:29. 10.1186/s13256-020-02582-8

7. Joo YB, Lim YH, Kim KJ, Park KS, Park YJ: Respiratory viral infections and the risk of rheumatoid arthritis . Arthritis Res Ther. 2019, 21:199. 10.1186/s13075-019-1977-9

8. Tang LV, Hu Y: Hemophagocytic lymphohistiocytosis after COVID-19 vaccination. J Hematol Oncol. 2021, 


\section{Cureus}

14:87. 10.1186/s13045-021-01100-7

9. Chang SE, Feng A, Meng W, et al.: New-onset IgG autoantibodies in hospitalized patients with COVID-19. Nat Commun. 2021, 12:5417. 10.1038/s41467-021-25509-3

10. Liu Y, Sawalha AH, Lu Q: COVID-19 and autoimmune diseases. Curr Opin Rheumatol. 2021, 33:155-62. 10.1097/BOR.0000000000000776

11. Jordan MB, Filipovich AH: Hematopoietic cell transplantation for hemophagocytic lymphohistiocytosis: a journey of a thousand miles begins with a single (big) step. Bone Marrow Transplant. 2008, 42:433-7. 10.1038/bmt.2008.232

12. Vilaiyuk S, Sirachainan N, Wanitkun S, Pirojsakul K, Vaewpanich J: Recurrent macrophage activation syndrome as the primary manifestation in systemic lupus erythematosus and the benefit of serial ferritin measurements: a case-based review. Clin Rheumatol. 2013, 32:899-904. 10.1007/s10067-013-2227-1

13. Junga Z, Stitt R, Tracy C, Keith M: Novel use of rituximab in macrophage activation syndrome secondary to systemic lupus erythematosus. BMJ Case Rep. 2017, 2017 :bcr2017221347. 10.1136/bcr-2017-221347

14. Schnaubelt S, Tihanyi D, Strassl R, et al.: Hemophagocytic lymphohistiocytosis in COVID-19: case reports of a stepwise approach. Medicine (Baltimore). 2021, 100:e25170. 10.1097/MD.0000000000025170 